\title{
Searching for the Optimal Parameters of the Thick Steep Seams' Under-Level Development
}

\author{
Svetlana Kostyuk ${ }^{1}$, Nikolay Bedarev ${ }^{2}$, Oleg Lyubimov, ${ }^{3, *}$, and Nikolay Kovalyov ${ }^{2}$ \\ ${ }^{1}$ T.F. Gorbachev Kuzbass State Technical University, 650000 Kemerovo, Russia \\ ${ }^{2}$ T.F. Gorbachev Kuzbass State Technical University, Prokopyevsk branch, Department of technology \\ and complex mechanization of mining, 653033 Prokopyevsk, Russia \\ ${ }^{3}$ T.F. Gorbachev Kuzbass State Technical University, Department of information and automated \\ manufacturing systems, 650000 Kemerovo, Russia
}

\begin{abstract}
At the present time the problem to search for variants for the development of steep thick coal seams is highly interest. Here there are, for example, variants for managing the workings' roof by caving or laying the worked out space. The authors proposed a number of methods for it, which are protected by patents. The permissible span of the roof exposure is estimated as a function of the type of the immediate roof of the mine, its thickness, bulk density and tensile strength, and the height of the underlevel is also determined. The technical result is provided by the presence of the protective inter-horizon strips and inter-sectional pillars, by the order of working out the excavation site and by the value of the step of the binding of the worked out space. The above solutions are acceptable for the Prokopyevsk-Kiselevsk coal region in Kuzbass.
\end{abstract}

\section{Introduction}

Technologies of the thick steep seams' development are characterized by practically ful lack of mechanization, high labor intensity, low level of safety and significant losses of coal in the bowels.

In this work the known variants of the thick steep seams' development by under-level systems with roofing control [1] and with binding of the established space [2], for which patents are available, are represented.

After analyzing the work [3] and with the substantiation of the roofing control systems' parameters, the patent [4], describing of this development system, was received.

The main difference between works [2,4] and work [1] is that there are furnaces between sub-levels and compensating gates dividing sub-levels into mining blocks with slope parallel to angle of formation of caved ground in mined area relatively to the line of bed course. And ventilation roadway for ventilating the below sub-level is made after mining and forced falling of the roof at above sub-level, note that above the ventilation roadway there maintained is a protective pillar that temporary prevents rock falling. In below sublevel the roof is controlled by stepped bypassing of caved ground from the above sub-level

* Corresponding author: oleglyub@gmail.com 
to bottom-hole area of below sub-level, exposed for allowable passage, note that allowable passage of roof exposure in bottom-hole area of below sub-level and corresponding extended distance $l_{\text {lim }}$ between the blocks in comparison to the above sub-level is defined by the expression:

$$
l_{\text {lim }}=l_{\text {calc }} C
$$

where $l_{\text {calc }}$ is the length value of the permissible span of exposure of the roof, determined by the known formulas, $\mathrm{m} ; C$ is the correction factor, taking into account the carrying capacity of the array of the overflow from the above sub-level, $\mathrm{C}$ always more than 1,0.

Fundamental conclusions and parameters are contained in $[5,6]$.

\section{Theory of the question}

Based on the analysis of the works [7-12] "Method of under-level plasts' development processing with the binding of the established space" was developed and patent was received.

The developed method is illustrated by the drawings, where fig. 1 shows a view along the seam strike line, fig. 2 shows a fragment of a section of the seam across the strike (section A-A).

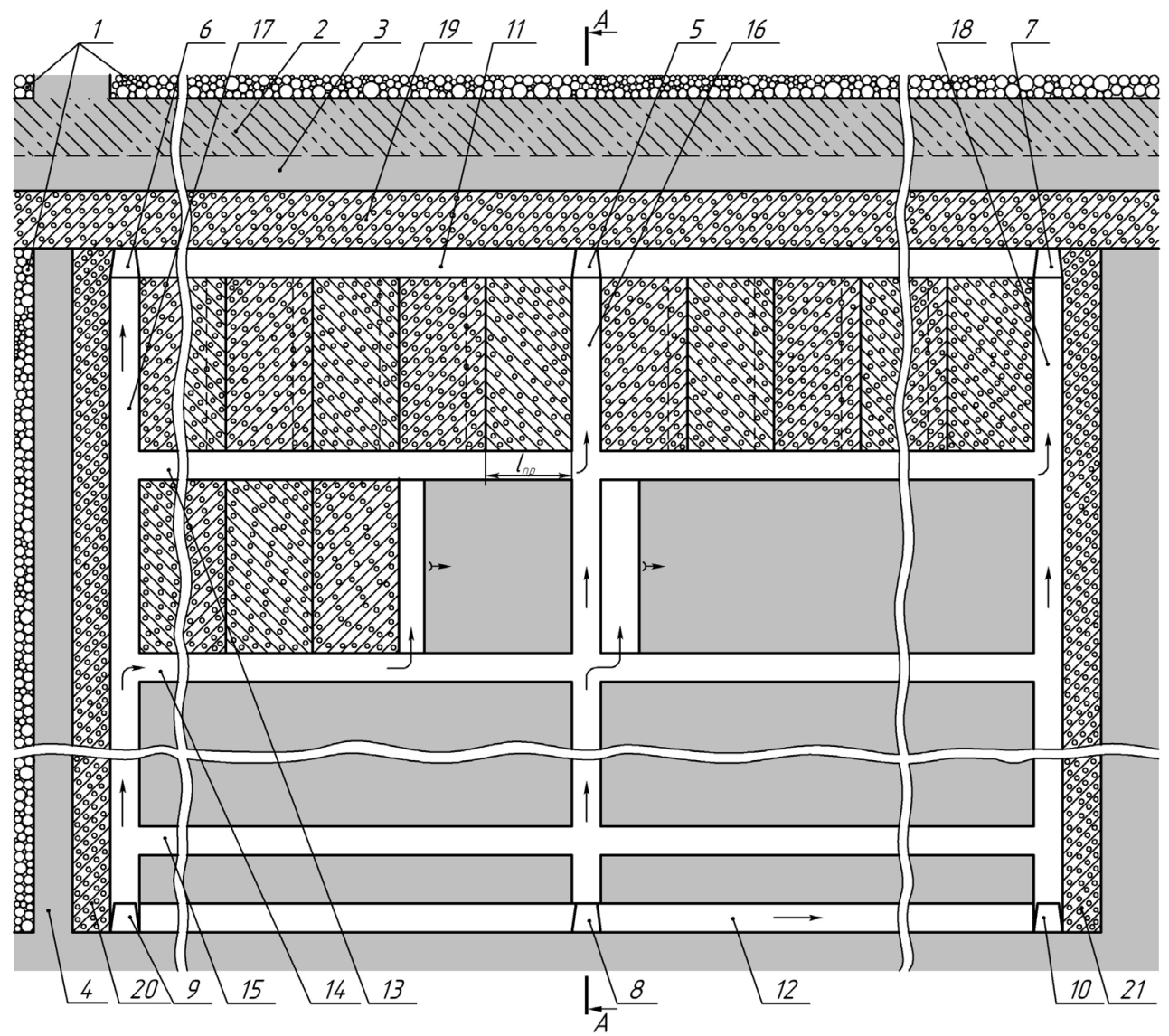

Fig. 1. View along seam strike line. 


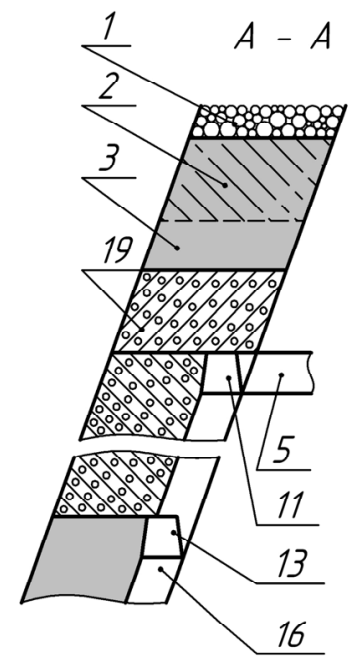

Fig. 2. Section of the seam across the strike (section A-A).

Preparation of the excavation site for working out is to:

- restrict preliminarily the field of the excavation site on the level to be worked: above, from the worked-out excavation sites 1 of the overlying worked level - by the inter-level cracked coal pillar 2 and by the inter-level coal pillar 3 without cracks; on the left, also from the worked-out excavation section 1 - by inter-segment coal pillar 4 (fig. 1, 2);

- conduct central 5 and flank 6, 7 cutouts on the ventilation horizon, central 8 and flank 9, 10 crossheads on the downill horizon, ventilation 11 and main 12 drifts, sub-level drifts 13 , 14, 15 and central 16 and flanking 17, 18 slopes (fig. 1).

The working out of the excavation site is carried out by sub-levels with short cleaning coalfaces ventilated at the expense of general depression.

Coal mining begins under the protection of the previously erected inter-horizon insulating strip 19 and in the presence of intercalation pillars also the insulating strips 20 and 21 that were previously erected simultaneously with the flank slopes 17 and 18 from the hardening tab with the advance of the cleaning work moving in the direction from the inter-horizon strip 19 to the main drift 12 . Wherein the working out of the excavating section is carried out in one wing - from the spent section towards the central slope, and in the other - from the central ramp to the target in the direction of the untreated excavation site, at a given value of the step of laying the worked-out space, no more than the allowable span of exposure of the roof of the formation along the line of incidence and strike to preserve the rock massif in a state near to natural.

Previous erection of the inter-horizon insulating strip 19, the erection of the insulating strips 20 and 21 simultaneously with the flank slopes 17 and 18 ensure the isolation of the spent horizon from aerodynamic permeability, reduce the probability of spontaneous combustion of coal, and, consequently, ensure the safety of mining operations, i.e. ensure the claimed technical result.

The roof appears as a plate, permanently supported on four sides, the span between the supports of which is constant in the fall of the formation, and along the strike are extended by short coalfaces, and the span should not exceed the permissible exposure by the condition of conservation of the rock massif in a state close to natural. The compliance with this condition is also ensured by the claimed technical result.

The order of development is predetermined by the condition that there are no the imposed reference pressures, as well as the absence of a possible deformation of the central ramp 16, which is ensured by the claimed technical result. In the wing on the left, where the working 
starts from the exhausted excavation site 1, the roof originally rests on the fall: from above to the interhorizon insulating strip 19, from below - the untreated rear sight of the lower under-level; along the strike: on the left - on the insulating strip 20, on the right - on the receding pillar as it is being worked off. In the wing to the right, where the working begins from the central ramp 16, the roof originally rests on the fall: from above - to the interhorizon insulating strip 19, from below - the untreated rear sight of the lower underlevel; along the strike: to the left - to the untreated left-wing pillar, to the right - on the receding pillar as it is being worked off.

The work on binding of the worked-out space, as the short cleaning faces are moved, is carried out in stages by the hardening tab. In this case, the worked-out space is laid with a step no more than the permissible span of exposure of the roof of the formation along the strike line, which can be determined from the well-known formula:

$$
l_{\text {roof }}=\sqrt{\frac{k \cdot \sigma_{\text {ext }} \cdot h_{\text {roof }}^{2}}{\left(1-\mu^{2}\right) \cdot q}} \mathrm{~m},
$$

where $k$ - coefficient taking into account the ratio of the lengths of sides of the exposure of the roof $a / b ;(k=2,0$ when $a / b=2,5)$;

$\sigma_{\text {ext }}$ - ultimate tensile strength of the immediate roof, $\mathrm{tn} / \mathrm{m}^{2}$;

$h_{\text {roof }}$ - thickness of the immediate roof, $\mathrm{m}$;

$\mu$ - Poisson's ratio;

$q$ - bulk mass of the immediate roof, $\mathrm{tn} / \mathrm{m}^{3}$;

\section{Results and Discussion}

According to the "Kiselevskaya" mine of the Prokopyevsk-Kiselevsk coal region, the required values are:

1) for IV Internal seam, with large and medium-grained sandstone as immediate roof, $\sigma_{\text {ext }}=8.0 \mathrm{MPa}=8.0 \times 10^{6} \mathrm{~N} / \mathrm{m}^{2}, h_{\text {roof }}=135 \mathrm{~m}, q=33.345 \times 10^{6} \mathrm{~N} / \mathrm{m}^{2}, \mu=0,33$

$$
l_{\text {roof }}=\sqrt{\frac{2 \cdot 8.0 \cdot 13.5^{2}}{\left(1-0.33^{2}\right) \cdot 33.345}}=9.91 \mathrm{~m},
$$

than the height of the sub-level along the line of incidence from the adopted relation $a / b=2.5$ will be $h=2.5 l_{\text {roof }}=2.5 \cdot 9.91=24.8 \approx 25 \mathrm{~m}$;

2) for III Internal seam, with mudstone as immediate roof, $\sigma_{\text {ext }}=4.0 \mathrm{MPa}=4.0 \times 10^{6} \mathrm{~N} / \mathrm{m}^{2}$, $h_{\text {roof }}=7.0 \mathrm{~m}, q=13.725 \times 10^{6} \mathrm{~N} / \mathrm{m}^{2}, \mu=0,22$

$$
l_{\text {roof }}=\sqrt{\frac{2 \cdot 4.0 \cdot 7.0^{2}}{\left(1-0.22^{2}\right) \cdot 13.725}}=5.48 \mathrm{~m}
$$

the height of the sub-level along the line of incidence from the adopted relation $a / b=2.5$ will be $h=13.7 \approx 14 \mathrm{~m}$;

3) for II Internal seam, with large and medium-grained aleurolite as immediate roof, $\sigma_{\text {ext }}=4.7 \mathrm{MPa}=4.7 \times 10^{6} \mathrm{~N} / \mathrm{m}^{2}, h_{\text {roof }}=9.0 \mathrm{~m}, q=15.75 \times 10^{6} \mathrm{~N} / \mathrm{m}^{2}, \mu=0,27$

$$
l_{\text {roof }}=\sqrt{\frac{2 \cdot 4.7 \cdot 9.0^{2}}{\left(1-0.27^{2}\right) \cdot 15.75}}=7.22 \mathrm{~m},
$$

the height of the sub-level along the line of incidence from the adopted relation $a / b=2.5$ will be $h=18.1 \approx 18 \mathrm{~m}$.

The mining in the coalface depending on the thickness of the seam can be carried out by harvesters (of the "Temp" type), drilling and blasting (drilling with blastholes along the 
strike, or long holes at the height of the substage), hydraulic softening of the massif, plowing units.

During the working out the next sub-stage, the role of the interhorizon insulating strip 19, as a support for the roof, fulfills the above-formed packing massif of the upper sub-level. So, the claimed technical result is provided.

Transportation of the broken coal is carried out downward along slopes 16, 17, 18 and further through the main drift 12 of the downhill horizon to the loading complex of the mine.

Ventilation of the coalfaces is carried out at the expense of general depression through the central crosscut 8 and flank crossheads 9 and 10, the central ramp 16 and flanking ramps 17 and 18 , the sub-level gates $13,14,15$ according to fig. 1 .

The principle of supporting the roof adopted in the proposed technical solution makes it possible to completely avoid, when preparing the site to work out, the need to leave the inter-level cracked coal pillar 2 and the inter-level coal pillar 3 without cracks on the left, as well as on the inter-stage coal pillar 4 , which allows to substantially reduce coal losses during mining, to increase the length of the cutting section along the strike (the distance between the flank cross-cuts 6 and 7), as well as the claimed technical result.

\section{Conclusion}

Thus, the claimed technical result is provided due to the facts that:

1) the working out of the formation begins under the protection of the previously erected inter-horizon insulating strip, and in the presence of intercalation pillars also the insulating strips that were previously erected simultaneously with the flank slopes from the hardening tab with the advance of the cleaning work moving in the direction from the inter-horizon strip to the main drift;

2) the working out of the excavating section is carried out in one wing - from the spent section towards the central slope, and in the other - from the central ramp to the target in the direction of the untreated excavation site;

3) the value of the step of laying the worked-out space, no more than the allowable span of exposure of the roof of the formation along the line of incidence and strike is given in order to preserve the rock massif in a state near to natural.

\section{References}

1. Coal Mining Methods (CMUT, Colorado, 2011)

2. V. Klishin, S. Klishin, Journ of Min. Sc., 46, 2 (2010)

3. S. Jayanthu, Strata control problems of underground coal mining Vis-à-vis geotechnical instrumentation and numerical model studies (DTSD, Berlin, 2011)

4. Pat. RF 2477795 (2013)

5. V. Miroshnikov, A. Gladir, Proc. of 4th Rus.-Chin. Sc. Forum, 21 (2014)

6. S. Kostyuk, N. Bedarev, O. Lyubimov, A. Shaikhislamov, E3S Web of Conf., 15, 01009 (2017)

7. S. Kostyuk, N. Bedarev, O. Lyubimov, A. Shaikhislamov, E3S Web of Conf., 21, 01002 (2017)

8. T. Zhao; Z. Zhang; Y. Yin; Y. Tan; X. Liu, J. S. Afr. Inst. Min. metall., 1, 118 (Johannesburg, 2018)

9. W. Jin-hui, Frontiers of Engineering Management (CMUT, Colorado, 2016) 
10. Hole Building Design Guide (CMUT, Colorado, 2016)

11. O. Kazanin, A. Sidorenko, V. Sementsov, Bul. NMU, 6 (2014)

12. F. Cui, 47th U.S. Rock Mech.Geomech. Symp.m 24, 56-62 (2013) 